\title{
Téoros
}

Revue de recherche en tourisme

\section{0 ans de recherche en tourisme}

\section{Alain A. Grenier}

Volume 30, numéro 1, 2011

URI : https://id.erudit.org/iderudit/1012102ar

DOI : https://doi.org/10.7202/1012102ar

Aller au sommaire du numéro

\section{Éditeur(s)}

Université du Québec à Montréal

\section{ISSN}

0712-8657 (imprimé)

1923-2705 (numérique)

Découvrir la revue

\section{Citer ce document}

Grenier, A. A. (2011). 30 ans de recherche en tourisme. Téoros, 30(1), 3-8.

https://doi.org/10.7202/1012102ar d'utilisation que vous pouvez consulter en ligne.

https://apropos.erudit.org/fr/usagers/politique-dutilisation/ 


\section{0 ans de recherche en tourisme}

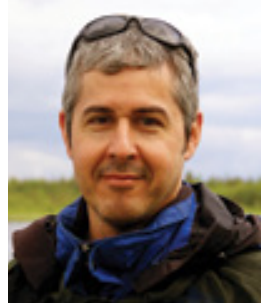

Alain A. GRENIER, Ph.D.

Directeur et rédacteur en chef de Téoros, depuis 2008

Professeur au Département d'études urbaines et touristiques Université du Québec à Montréal (ESG-UQAM)

grenier.alain@uqam.ca

Il n'y pas si longtemps, les étudiants et les chercheurs francophones qui voulaient participer au développement de la connaissance universitaire en tourisme n'avaient d'autre choix que de se tourner vers le monde anglo-saxon : c'est là que se trouvaient principalement les périodiques de recherche en tourisme. Tant pour le lecteur à l'affût des derniers résultats de recherche que pour l'universitaire voulant publier les résultats de ses travaux, il n'y avait pas d'autre option que les publications étrangères. En français, il n'existait rien. Quand on connaît l'importance pour les chercheurs (j'entends ici les professeurs, les consultants et chargés de projets, les doctorants et autres étudiants de cycles supérieurs) de participer à l'édifice de leur domaine, le partage de la connaissance passe bien sûr par l'existence d'outils et de véhicules de transmission de la science - mais encore faut-il pouvoir travailler dans sa langue.

La revue Téoros que vous tenez entre vos mains est cet outil de travail unique consacré à la recherche universitaire en tourisme, en français. Cette revue est le fruit de trente ans d'efforts soutenus déployés par des gens audacieux qui se sont succédé et qui ont, chacun à leur façon, contribué à faire de Téoros l'outil de recherche et de développement unique qu'il est devenue au fil du temps.

Rappelons que Téoros a germé de l'initiative de trois professeurs du Département d'études urbaines et touristiques (École des sciences de la gestion, Université du Québec à Montréal) en 1982. Louis Jolin, Marc Laplante et Jean Stafford produisent alors des "cahiers de recherche» en tourisme (voir encadré page suivante). L'objectif de ces cahiers est de contribuer, en l'absence d'outils adéquats, à l'augmentation des connaissances sur le tourisme (Jolin, $1982: 2$ ). Les cahiers cherchent ainsi à se distinguer des médias traditionnels, qui abordent (encore aujourd'hui) le tourisme à des fins de promotion — faire connaître et vendre des destinations. Ce point est fondamental. Téoros n'est pas l'outil de promotion des destinations, mais bien celui de l'analyse et de la compréhension des phénomènes touristiques. On reproche beaucoup à l'industrie touristique son caractère de masse, envahisseur, pollueur des environnements fragiles et contaminant des cultures. On ne peut corriger les faiblesses du tourisme (en améliorer la gestion) sans comprendre les phénomènes du tourisme en profondeur. Qui sont les touristes, qu'est-ce que l'expérience touristique, comment interagissent les différents acteurs, voilà les premières questions auxquelles on ne fait que commencer à répondre.

Le premier cahier de Téoros (du grec «celui qui voyage », francisé, avec son accent grave, pour les besoins de la revue) prend son envol en février 1982 (illustration 1).

Les premiers numéros sont plutôt des réflexions sur le développement du tourisme au Québec. Le développement de la revue accompagne en partie celui de son département, à l'UQAM. En 1987, le département, jusqu'alors centré sur les études urbaines, consacre le rôle joué par les études touristiques depuis 1978 au sein de l'unité, qui prend alors le nom de Département d'études urbaines et touristiques (DEUT). Téoros grandit de ces changements et commence à s'imposer comme
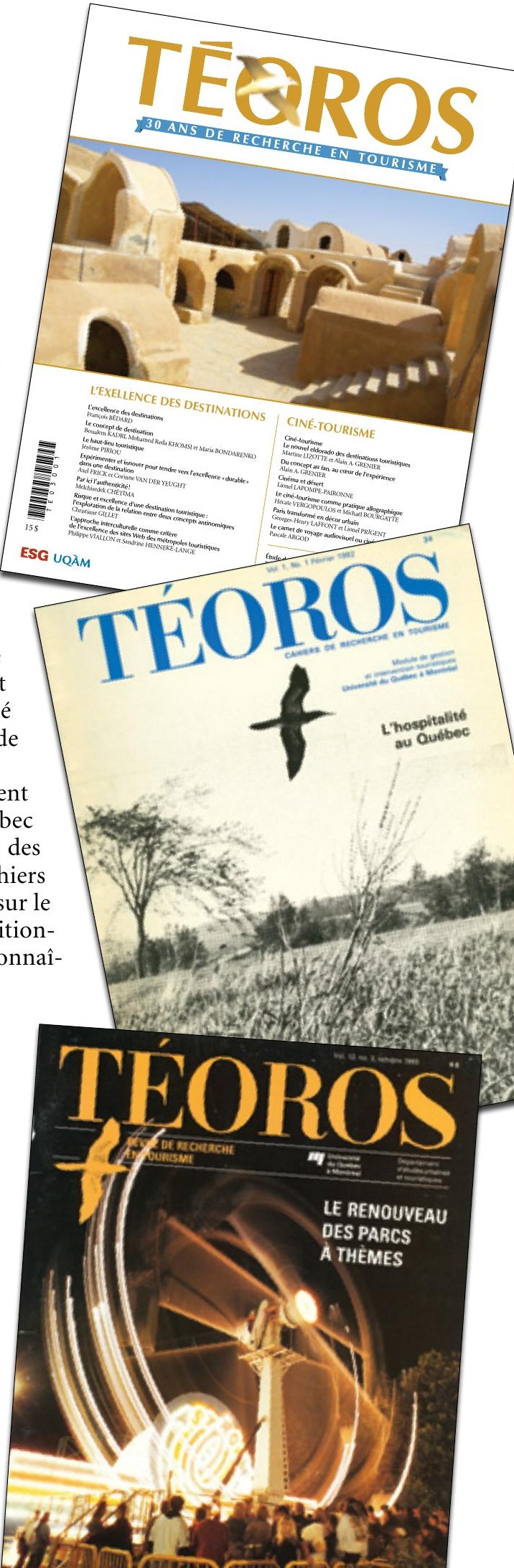



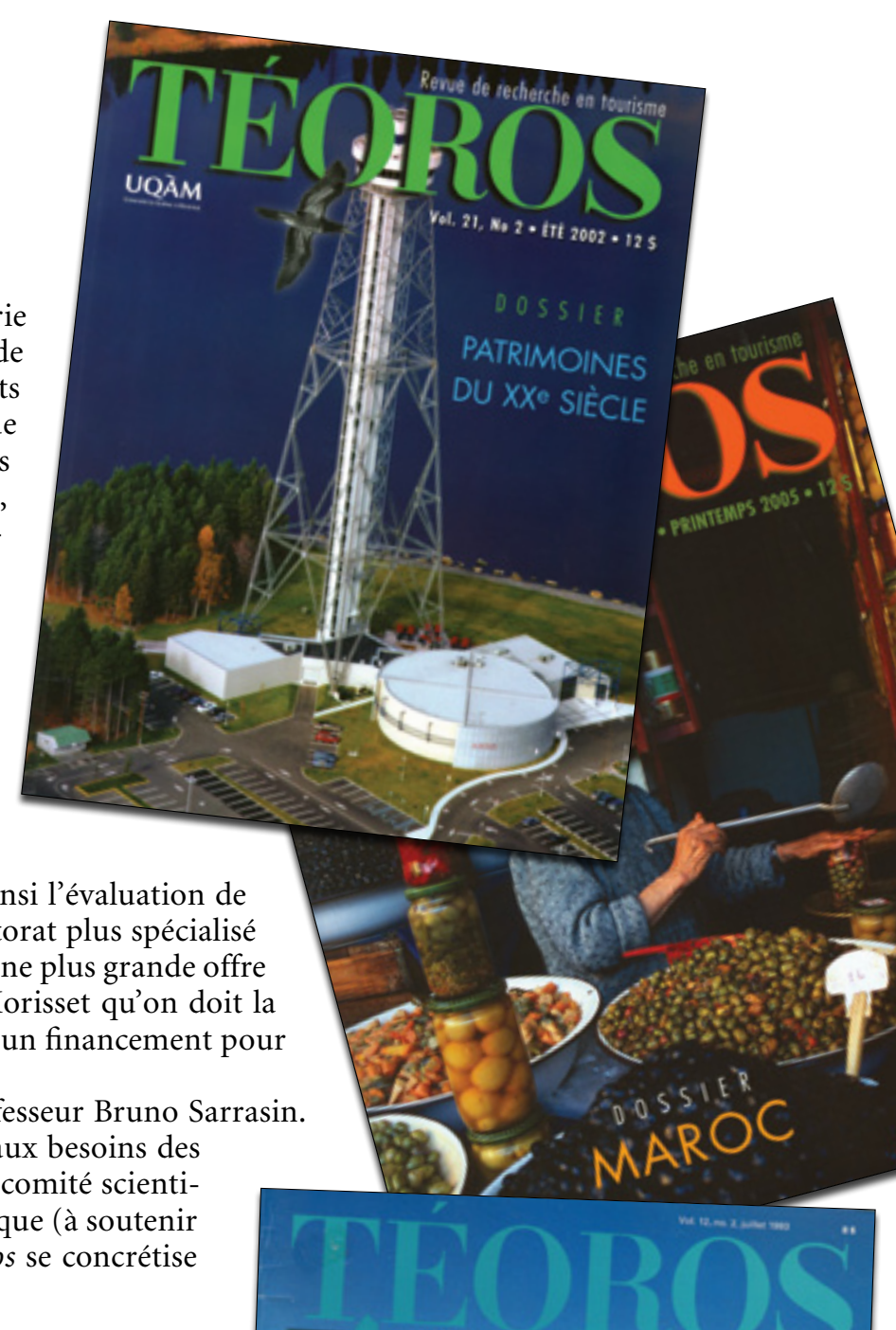

\section{De l'adolescence à la maturité}

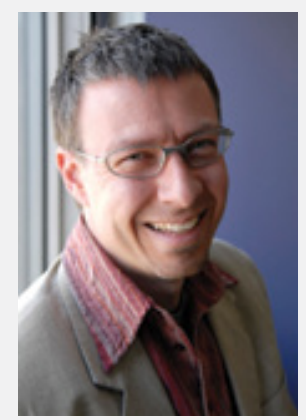

J'ai occupé les fonctions de rédacteur en chef de l'été 2003 au printemps 2008. Le virage scientifique de Téoros avait été amorcé sous la direction de la professeure Lucie K. Morisset, mais le comité de rédaction n'était pas encore prêt à voir la revue passer de la mission de "transfert», qui avait marqué sa fondation, vers celle de la production de savoirs répondants aux critères de validité scientifique. Voilà le principal défi ayant marqué mes cinq années de direction : accomplir le virage de l'adolescence - que la direction précédente avait géré avec brio - à la maturité — sur laquelle la direction suivante allait capitaliser avec une grande efficacité.

Le maintien d'une grande diversité de thèmes ancrés sur les enjeux de l'actualité (risques politiques, qualité, patrimoine, gastronomie, etc.) et la création de chroniques d'analyses portées, à partir de 2003, par la Chaire de recherche du Canada en patrimoine urbain et la Chaire de tourisme Transat, et par le Centre international de formation et de recherche en tourisme, ont permis de garder un lien fort avec les besoins de l'industrie touristique. La mobilisation du comité de rédaction, le nombre et la qualité des articles parus dans la section «arbitrée» de la revue et la création d'un comité scientifique à partir du premier numéro de 2007 consacreront la nouvelle mission de Téoros. Il faudra cependant attendre le premier numéro du volume 28 (2009) pour que cette transition soit pleinement consommée.

Bruno SARRASIN, Ph.D.

Directeur et rédacteur en chef de Téoros, de l'été 2002 au printemps 2008 Professeur au Département d'études urbaines et touristiques (ESG-UQAM) Directeur des programmes de $1^{\mathrm{er}}$ cycle en gestion du tourisme et de l'hôtellerie Directeur de la collection Tourisme aux Presses de I'Université du Québec Membre du comité de direction et de rédaction de Téoros 
apportés à Téoros, que j'incarne, à titre de premier directeur du nouveau Téoros. Il faudra un peu plus de deux ans pour faire accepter cette renaissance de la revue. S'il en revient à chacun de juger les résultats de l'entreprise, soulignons que Téoros reçoit en moyenne pour chaque thématique deux à trois fois plus d'articles qu'auparavant, se donnant ainsi le choix de la qualité. Nos rédacteurs viennent des quatre coins de la planète, du Québec et de France, bien entendu, mais même d'aussi loin qu'Israël ou la Nouvelle-Zélande. Nous ne publions pas que les écrits des chercheurs : plusieurs doctorants trouvent leur compte dans Téoros, en y publiant parfois les premiers articles de leur carrière. Nos pages comptent aussi des textes de consultants, qui apportent au cadre théorique désormais nécessaire des cas concrets en matière de gestion (durable) du tourisme.

\section{L'envers du décor}

La réalisation de Téoros n'est pas qu'une affaire de recherche et d'écriture. Avant que nos appels à textes ne soient lancés, il faut d'abord choisir, en collaboration avec le comité de direction et de rédaction (voir encadré ci-contre), les thématiques à être développées dans nos pages. Il faut aussi solliciter des rédacteurs invités qui parraineront les dossiers thématiques. Environ six mois après avoir lancé nos appels à textes, direction et rédacteurs invités étudient les propositions reçues. Toutes celles qui respectent la politique de rédaction de Téoros (voir en ligne : http://teoros.revues.org/168) sont soumises au processus d'évaluation anonyme par les pairs. Les textes acceptés par les évaluateurs sont ensuite retournés à leurs auteurs aux fins de correction sur le fond. S'ajouteront encore au travail de l'équipe la révision linguistique et le montage graphique des textes. Il faut compter aussi plusieurs séances de révisions avec l'équipe graphique. Plus de 16 mois s'écouleront ainsi en moyenne entre la soumission des manuscrits et leur transposition en articles dans la revue.

Outre le travail avec les auteurs, la gestion de Téoros nécessite parallèlement de voir à son financement. De par sa spécialisation, Téoros n'est pas une revue grand public. Ainsi, son financement, comme celui de tous les périodiques scientifiques, demeure davantage une affaire de bon vouloir et de bons principes des partenaires. Néanmoins, avec un budget modeste et des partenaires fidèles (le ministère du Tourisme du Québec, Tourisme Montréal) et le soutien de l'Université (l'École des sciences de la gestion, la Chaire de recherche du Canada en patrimoine urbain, la Chaire de tourisme Transat et le Centre international de formation et de recherche en tourisme), Téoros tient la barre depuis maintenant 30 ans. Il importe aussi de souligner que la direction de la revue est entièrement assumée par les professeurs nommés au poste par le DEUT et qui ne touchent aucune rémunération pour ce travail de tous les instants.

\section{Et demain?}

Notre dernière grande transformation se vit en ce moment dans l'univers de l'électronique. Téoros adhère depuis 2009 au portail revues.org. La mise en ligne nous permet d'abord de partager nos archives de ces 30 années d'expertise avec un lectorat désormais composé de milliers de lecteurs à travers le monde. Ce choix de plate-forme, nous l'avons aussi fait en fonction de notre philosophie d'accès gratuit à l'information. Moyennant une barrière de deux ans, nos articles deviennent par la suite libres d'accès, en ligne. À quoi bon la version papier alors? Parce que, quoi qu'on en dise, nous croyons encore à l'expérience de la lecture sur papier, du crayon marqueur pour les passages plus importants. On a dit de l'arrivée de la télévision qu'elle tuerait le cinéma, que l'ordinateur tuerait le livre, que l'avion remplacerait le navire. Il n'en est rien. Une nouvelle invention ne remplace pas l'ancienne; elle vient la compléter. 
Notre avenir sera donc fait d'électronique, mais aussi de papier. Téoros continuera d'innover avec, déjà en préparation pour les prochains numéros, des thématiques pleines d'originalité. Je souhaite compléter ce texte anniversaire en remerciant les gens qui m’appuient dans mon travail de directeur et rédacteur en chef depuis maintenant plus de trois ans, en commençant par mes adjoints, Barbara Julien, Mathieu Dormaels, Juliana Chavez dos Santos et Ari Virtanen, ainsi que tous ceux et celles qui les ont précédés depuis 2002. Ces adjoints ont vécu tour à tour la naissance de chacune des pages de la revue - avec les angoisses et les plaisirs que cela comporte. Je souligne aussi le travail exceptionnel de notre équipe graphique, Marie-Claude Béland et Alain Bolduc, de B Graphistes, sans qui Téoros n'aurait pas la même classe. Je remercie aussi mon comité de direction, Paul Arsenault, Gérard Beaudet, Normand Cazelais, Louis Jolin, Boualem Kadri, Marie Lequin, Jan Lundgren et Bruno Sarrasin, pour son travail et son soutien. Le comité scientifique n'est pas en reste; son travail se fait dans l'ombre, mais il n'en est pas moins essentiel à la revue et à son bon fonctionnement. Merci aussi aux auteurs et aux évaluateurs, qui contribuent, de numéro en numéro, à faire de Téoros une revue essentielle en recherche.

Merci enfin à vous, chers lecteurs et lectrices. Et merci tout particulièrement aux abonnés, qui, par leur intérêt et leur fidélité renouvelée, sont finalement les gardiens de Téoros et de sa pérennité.

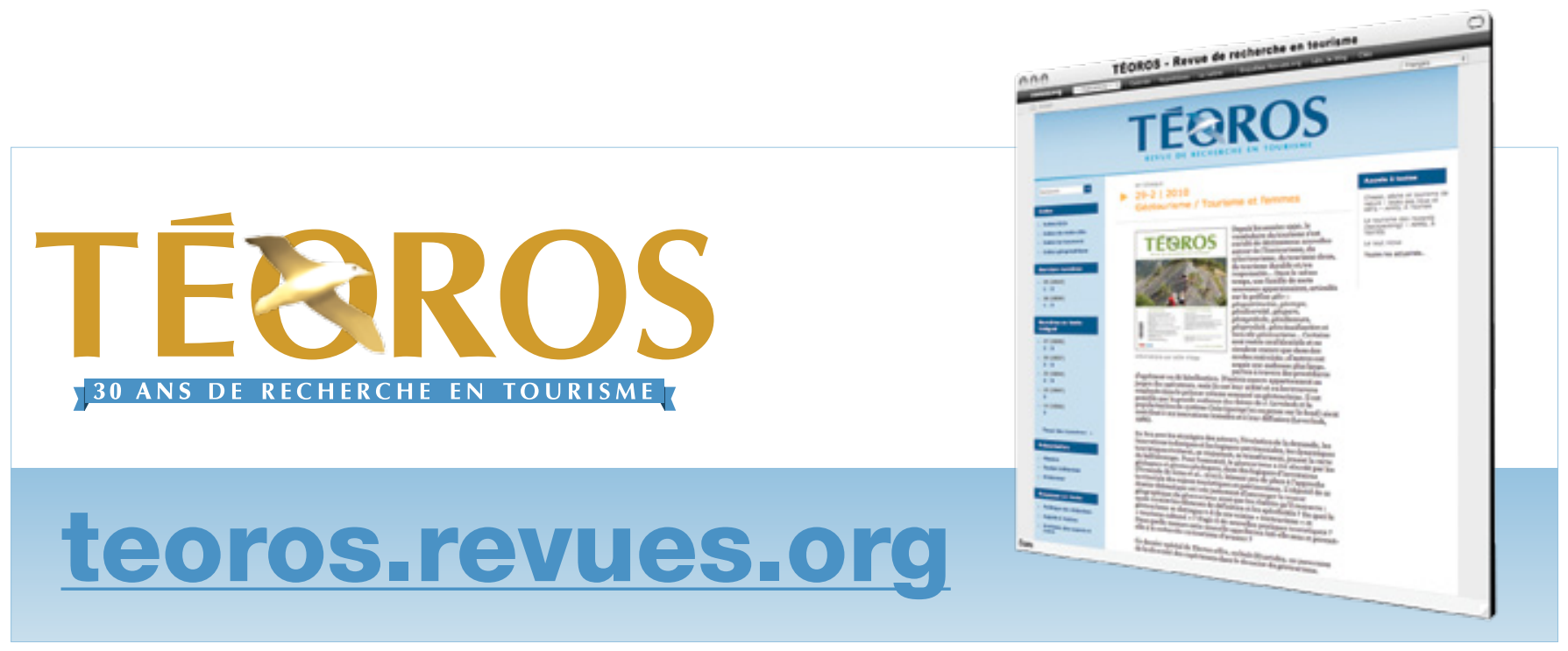

